\title{
Low-dose dobutamine cardiac magnetic resonance imaging with myocardial strain analysis predicts myocardial recoverability after coronary artery bypass grafting
}

\author{
D. Dean Potter, MD, ${ }^{\text {a }}$ Philip A. Araoz, MD, ${ }^{\text {b }}$ Kiaran P. McGee, PhD, ${ }^{b}$ W. Scott Harmsen, MS, ${ }^{c}$ \\ Jayawant N. Mandrekar, $\mathrm{PhD}{ }^{\mathrm{c}}$ and Thoralf M. Sundt, III, MD ${ }^{\mathrm{a}}$
}

From ${ }^{a}$ the Division of Cardiovascular Sur-

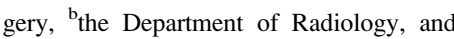
cthe Division of Biostatistics, Mayo Clinic and College of Medicine, Rochester, Minn.

Supported by grants T32-HL-07776 and R01-HL62291-04 from the National Heart, Lung, and Blood Institute.

Received for publication June 27, 2006; revisions received Dec 29, 2007; accepted for publication Jan 5, 2008

Address for reprints: Thoralf M. Sundt, III, MD, Division of Cardiovascular surgery, Mayo Clinic, 200 First St SW, Rochester, MN 55905 (E-mail: johnson.barbara4@ mayo.edu)

J Thorac Cardiovasc Surg 2008;135:1342-7 $0022-5223 / \$ 34.00$

Copyright $(\subset 2008$ by The American Association for Thoracic Surgery

doi:10.1016/j.jtcvs.2008.01.005
Objectives: Accurate prediction of recovery of dysfunctional myocardium would optimize risk/benefit analysis among patients with coronary artery disease and decreased ventricular function. Tissue-tagged magnetic resonance imaging permits quantitative assessment of changes in ventricular function and may improve the prediction of myocardial recovery after coronary artery bypass grafting.

Methods: Thirteen patients underwent preoperative and postoperative stress magnetic resonance imaging with strain analysis at rest with 5 and $10 \mu \mathrm{g} \cdot \mathrm{kg}^{-1} \cdot \mathrm{min}^{-1}$ dobutamine. Two-dimensional strain analysis was performed on a single midventricular short-axis image divided into 6 regions for each patient $(n=78)$. Regional minimum principal, circumferential, and radial strain values were calculated at each stress level. Regional changes in postoperative strain were correlated with changes in preoperative dobutamine stress by means of logistic regression. Receiver operating characteristic curves were created to determine the accuracy of preoperative dobutamine stress for the prediction of postoperative myocardial recoverability.

Results: Minimum principal, circumferential, and radial strain values at 5 and $10 \mu \mathrm{g}$ of dobutamine differed significantly from baseline strains $(P<.05)$. Receiver operator characteristic curves found minimum principal strain to be $75 \%$ accurate for prediction of recoverability at both stress levels. Circumferential strain was $72 \%$ and $70 \%$ accurate at 5 and $10 \mu \mathrm{g}$, respectively, whereas radial strain was $77 \%$ and $64 \%$ accurate at 5 and $10 \mu \mathrm{g}$, respectively.

Conclusions: Dobutamine-stressed tissue-tagged magnetic resonance imaging with strain analysis is feasible to quantitatively predict myocardial recoverability after coronary artery bypass grafting. Further study is required to determine the optimal strain parameter for predicting myocardial recoverability after surgical revascularization.

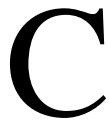
oronary artery bypass grafting $(\mathrm{CABG})$ can improve left ventricular $(\mathrm{LV})$ function in patients with LV dysfunction, resulting in fewer symptoms and increased survival. ${ }^{1,2}$ Distinction between reversible and irreversible myocardial injury with currently used techniques is imperfect. Improved accuracy in predicting recoverability could help tailor revascularization procedures to optimize patient benefits and reduce risks. Recently, magnetic resonance (MR) imaging with dobutamine stress has been used to assess functional and morphologic variables of myocardial viability by means of quantitative systolic wall thickening ${ }^{3-5}$ and visual assessment. ${ }^{6-8}$ Radiofrequency (RF) tissue tagging appears to improve the visual detection of wall motion abnormalities with myocardial ischemia ${ }^{9,10}$; however, 2-dimensional myocardial strain analysis has been used to quantify these variables. ${ }^{10}$ Myocardial strain parameters hold promise as highly accurate regional variables to quantitate functional changes, which might improve the prediction of myocardial recoverability after revascularization. 


\section{Abbreviations and Acronyms}

$\mathrm{CABG}=$ coronary artery bypass grafting

$$
\begin{array}{ll}
\mathrm{LV} & =\text { left ventricular } \\
\mathrm{MR} & =\text { magnetic resonance } \\
\mathrm{RF} & =\text { radiofrequency }
\end{array}
$$

The goal of this study was to evaluate the feasibility of using this technology clinically by comparing the predictive value for myocardial recovery after revascularization of 3 different MR myocardial strain parameters with dobutamine stress in patients undergoing CABG.

\section{Materials and Methods Study Patients}

This prospective study was approved by the Institutional Review Board at Mayo Clinic, Rochester. Informed consent was obtained from 20 patients with multivessel coronary artery disease and at least 1 regional wall motion abnormality, as determined by means of echocardiography or left ventriculography, scheduled for elective CABG. Patients with unstable angina, significant valvular disease, cardiac arrhythmia, or contraindication to MR imaging were excluded. Preoperative MR imaging with RF tissue tagging was performed the day before the operative procedure. Postoperative MR imaging was performed at least 3 months after the surgical procedure. Five short-axis tissue-tagged MR images were obtained at rest and at 2 levels of dobutamine stress. Images were acquired after 3 minutes of dobutamine infusion to obtain a stable state of drug at 5 and $10 \mu \mathrm{g} \cdot \mathrm{kg}^{-1} \cdot \mathrm{min}^{-1}$. Heart rate, continuous pulse oximetry, blood pressure, and near real-time assessment of regional wall motion abnormalities were recorded during preoperative and postoperative imaging. Dobutamine infusion was terminated when the target heart rate was reached, when development of angina pectoris associated with a new regional wall motion abnormality was determined by means of near real-time assessment, when cardiac arrhythmia developed, or on completion of the stress protocol. The total time for image acquisition at baseline and dobutamine infusion at 2 stress levels was less than 60 minutes. Oral $\beta$-blockers were not withheld before stress testing, and atropine infusion was not used to augment heart rate during testing.

\section{MR Imaging Acquisition}

The imaging technique has been previously described. ${ }^{11}$ Images were acquired on a 1.5-T MR imaging system (Signa Excite; GE Medical Systems, Milwaukee, Wis). Gradient strength was 40 $\mathrm{mT} / \mathrm{m}$, and slew rate was $150 \mathrm{~T} / \mathrm{m} / \mathrm{s}$ in zoom mode. A phased-array torso coil was used. The pulse sequence was a 2-dimensional gradient echo sequence that was electrocardiographically gated and performed during breath holds. Using spatial modulation of magnetization, RF tissue tags were created in an orthogonal grid pattern of presaturation in the myocardium in the short axis. ${ }^{12}$ Slice thickness was $8 \mathrm{~mm}$, with a gap of $7 \mathrm{~mm}$, which allowed for acquisition of an image in the apex, mid, and base. Field of view varied by patient, with a range between 360 and $480 \mathrm{~mm}$. The remaining imaging parameters were as follows: matrix, $256 \times 128$; flip angle, $20^{\circ}$; number of excitations, 1 . Echo time was selected as minimum, which resulted in echo times that ranged between 1.4 and $5.2 \mathrm{~ms}$ (mean, $5.0 \mathrm{~ms}$ ). Repetition time was not manipulable by the operators but ranged between 8.7 and $8.8 \mathrm{~ms}$. The number of cardiac phases acquired per slice was 20 . In all patients, the total time required for patient imaging was less than 60 minutes.

\section{Conventional Indices of LV Function}

LV ejection fraction was calculated by using Mass Analysis Plus (Mass Analysis Plus; MEDIS Medical Imaging Systems, Leiden, The Netherlands) running on Dell workstations. The end-diastolic image was chosen as the first image in sequence, and the end-systolic image was identified as the image just before ventricular relaxation (smallest ventricular chamber). Endocardial and epicardial boundaries were manually outlined for each image. The papillary muscles, trabeculae, and epicardial fat were excluded from the analysis. Ejection fraction was determined for the entire left ventricle. A finite element model of the left ventricle was constructed, consisting of 6 quadrilateral elements corresponding to the anteroseptal, anterior, anterolateral, posterolateral, posterior, and posteroseptal walls by placing an axis point at the intersection of the anterior right ventricular endocardium and LV epicardium (Figure 1).

\section{Measured and Predicted Displacements}

Identical images used for calculation of percentage thickening were analyzed with custom software running on Dell workstations. Endocardial and epicardial boundaries were manually identified for each image and represented by closed third-order B-spline curves. The

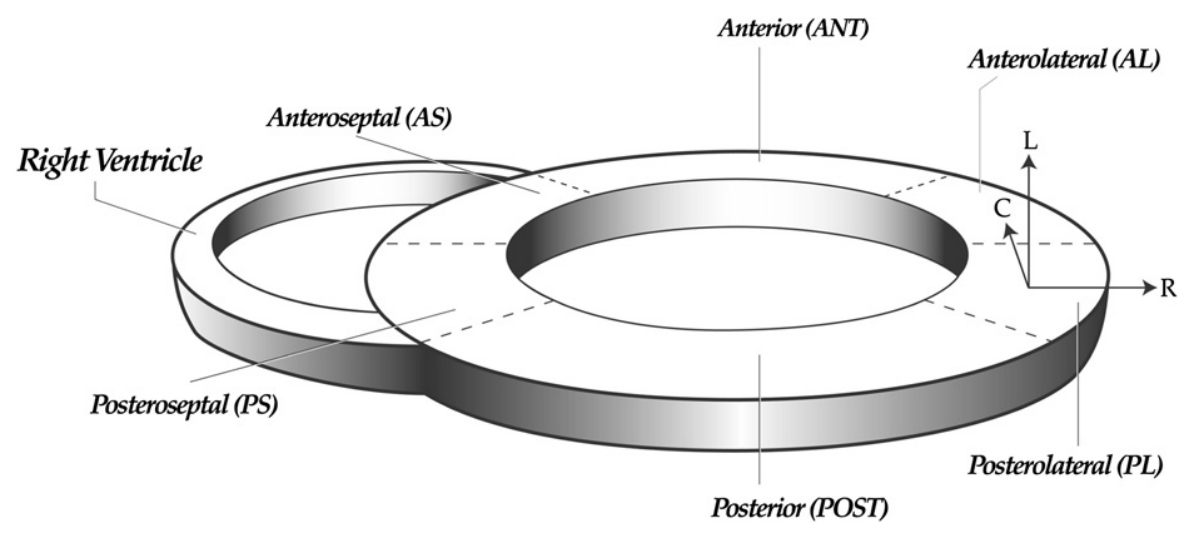

Figure 1. Schematic representation of the 6 ventricular regions. A vector diagram depicts the vector directions for circumferential (C) and radial (R) strains, longitudinal $(L)$. 
initial representation of the tissue tag lines was constructed from the end-diastolic image based on the known spacing $(0.8 \mathrm{~cm})$ between adjacent tag lines. Tag lines were located on successive images by using an automatic algorithm based on local pixel density. Tracking software overlaid a spline for each tissue tag until reaching end-systole. Two-dimensional systolic displacements were computed at each tag-line intersection within the myocardium. Regions of the left ventricle were constructed as previously defined for percentage thickening. By using a least-squares fitting model, predicted displacement information was calculated for any point within the myocardium from known measured displacements by using a finite element software package (StressCheck; ESRD, St Louis, Mo). The combination of known and predicted displacement information provided a continuous representation of displacement information for the entire ventricle. ${ }^{13}$

\section{Strain Analysis}

Strain is defined as the deformation of an object normalized to its original shape. Strain is a dimensionless quantity, and the resulting deformation of the material is expressed as either fractional or the percentage change from the original dimension. Strain $(\varepsilon)$ can be mathematically defined as follows: $\varepsilon=l-l 0 / l 0=\Delta l / l 0$, where $l$ is the instantaneous length, $l 0$ is the original length, and $\Delta l$ is the change in length. The original length refers to the end-diastolic muscle length. We examined 3 components of strain. Minimum principal stain is the minimum normal strain among all directions emanating from a particular point. The minimum principal strain is associated with the maximum shortening experienced by the tissue at a given point. Circumferential strain is the normal strain along a tangential vector emanating from the LV wall and is associated with contraction or relaxation in the short-axis plane. Radial strain represents normal strain along a perpendicular vector from the LV wall and is associated with wall thickening (Figure 1). By using least-squares fitting of the measured displacement data, average regional strain values were computed. Details of the p-version finite element formulation can be found in Szabo and Babuska. ${ }^{14}$

\section{Statistical Analysis}

Analysis was performed on 6 regions per patient, resulting in 78 ventricular regions in the 13 patients studied. Where applicable, mean strain values for each region were reported with standard deviations. Each region was considered to be an independent measure and divided into 2 groups: recoverable and not recoverable. Regions were defined as recoverable if the strain value improved from preoperative rest to postoperative rest. Univariate logistic regression analysis was used to assess the association between change in strain measurements for each stress level (rest to $5 \mu \mathrm{g}$ and rest to $10 \mu \mathrm{g}$ ) and recoverability of the myocardium in the ventricular region for each of the 3 strain parameters. Receiver operating characteristic analysis was performed for each of the strain/stress parameters of interest. The area under the receiver operating characteristic curve was estimated separately by using trapezoidal rule based on the predicted values from each of the univariate logistic regression models that were fitted. The area under the curve was used as a measure of discrimination. It is a measure of the likelihood that a patient who has recovered will have a higher probability than a patient who has not recovered. An area under the curve of 0.7 and greater is considered an acceptable discrimination. ${ }^{15}$

Cutoff points for the strain parameters at each stress level that determined recoverability of myocardium were selected such that the sum of sensitivity and specificity was maximal and had a sensitivity of $80 \%$ or $90 \%$. Sensitivities, specificities, and likelihood ratios corresponding to the identified cutoff points were reported, where likelihood ratio is calculated as a ratio of Sensitivity over (1-specificity). We also treated prechange to postchange resting strain values as a continuous outcome variable and analyzed data using linear regression. The significance of the clinical conclusions remained.

\section{Results}

\section{Patients}

Written informed consent was obtained from 20 patients for this study. Seven patients were excluded from the final analysis. Reasons for exclusion after obtaining informed consent

TABLE 1. Demographics and clinical characteristics

\begin{tabular}{|c|c|c|c|c|c|c|c|c|}
\hline \multirow[b]{2}{*}{ Patient no. } & \multirow[b]{2}{*}{ Age $(y) /$ sex } & \multicolumn{3}{|c|}{ Coronary disease } & \multirow[b]{2}{*}{$\beta$-blocker } & \multirow[b]{2}{*}{ DM } & \multirow[b]{2}{*}{ LVEF $(\%)$, before/after } & \multirow[b]{2}{*}{ EDV $(\mathrm{mL})$, before/after } \\
\hline & & LAD & Cx & RCA & & & & \\
\hline 1 & 65/Male & 100 & 70 & 100 & + & - & $15.7 / 16.5$ & $138 / 130$ \\
\hline 2 & 55/Male & 50 & 99 & 99 & + & + & $63.9 / 62.5$ & $67 / 78$ \\
\hline 3 & 61/Male & 70 & 70 & 40 & + & + & $28.0 / 26.2$ & $86 / 94$ \\
\hline 4 & 52/Male & 99 & 30 & 100 & + & - & $66.6 / 59.6$ & $83 / 61$ \\
\hline 5 & 67/Male & 90 & 80 & 90 & + & - & $63.9 / 61.8$ & $79 / 73$ \\
\hline 6 & 64/Female & 80 & 70 & 100 & - & + & $51.8 / 49.3$ & $62 / 46$ \\
\hline 7 & 58/Female & 70 & 50 & 99 & + & + & $54.5 / 44.7$ & $59 / 65$ \\
\hline 8 & 59/Male & 70 & 40 & 95 & + & - & $23.4 / 23.4$ & $159 / 83$ \\
\hline 9 & 56/Male & 80 & 80 & 90 & + & - & $57.1 / 51.6$ & $78 / 100$ \\
\hline 10 & $60 /$ Female & 70 & 100 & 100 & + & - & $67.1 / 71.4$ & $51 / 44$ \\
\hline 11 & 43/Male & 30 & 100 & 100 & + & + & $38.0 / 42.6$ & $69 / 80$ \\
\hline 12 & 61/Male & 100 & 70 & 80 & + & - & $50.1 / 51.0$ & $84 / 74$ \\
\hline 13 & 62/Male & 70 & 70 & 100 & + & - & $16.4 / 24.1$ & $126 / 93$ \\
\hline
\end{tabular}

$A D$, Left anterior descending coronary artery; $C X$, left circumflex coronary artery; $R C A$, right coronary artery; $D M$, diabetes mellitus; VEF, left ventricular ejection fraction; $E D V$, end-diastolic volume. 

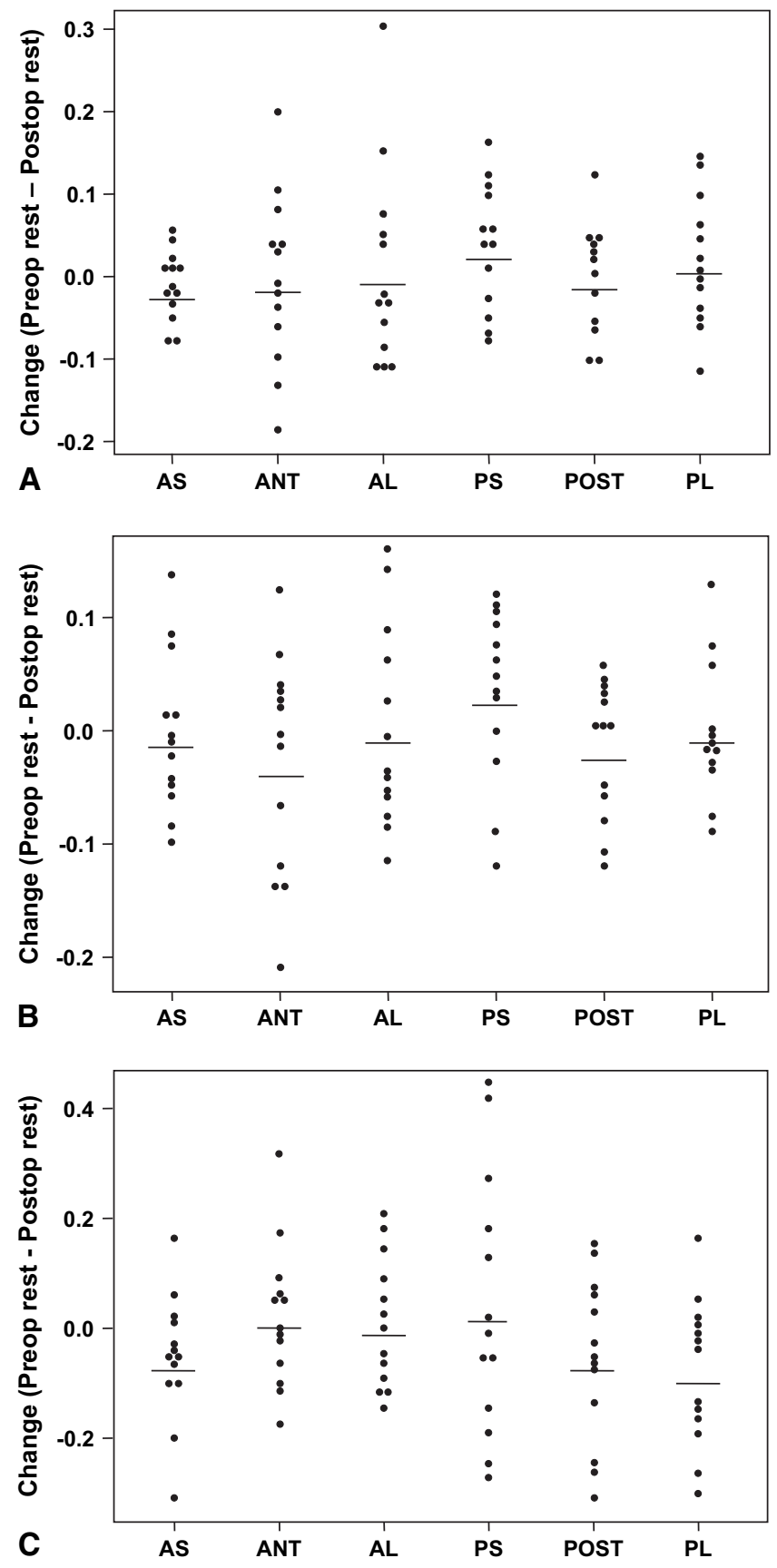

Figure 2. Postoperative change in myocardial function by myocardial strain measurements. $A S$, Anteroseptal; $A N T$, anterior; $A L$, anterolateral; $P L$, posterolateral; $P O S T$, posterior; $P S$, posteroseptal. The horizontal bar indicates mean change. The $P$ value was determined by using paired Student $t$ tests. A, Regional change for minimum principal strain. The change in regional minimum principal strain was not significantly different after coronary artery bypass. $B$, Regional change for circumferential strain. The change in regional circumferential strain was not significantly different after coronary artery bypass. C, Regional change for radial strain. The change in regional radial strain was not significantly different after coronary artery bypass. included inability to gate in 1 patient, MR scanner malfunction in 1 patient, poor imaging quality in 1 patient, bypass graft occlusion requiring graft revision in 1 patient, patient refusal of operative intervention in 1 patient, patient refusal of follow-up imaging because of claustrophobia in 1 patient, and patient refusal of dobutamine stress testing in 1 patient. Demographic and clinical information for the final study group of 13 patients are summarized in Table 1. All operative procedures were performed with cardiopulmonary bypass and high potassium cardioplegia infused every 20 minutes. The mean number of bypass grafts was $3.6 \pm 0.8$. No study patient experienced postoperative complications. The mean number of months to postoperative images was $4.0 \pm 1.5$. One patient delayed postoperative imaging by 8.6 months because of travel plans. Five patients had improved LV ejection fraction, and 7 patients had reduced LV end-diastolic volumes postoperatively (Table 1). Overall, global strain values after revascularization were similar to preoperative values (Figure 2).

Dobutamine-stressed Myocardial Strain Analysis and Prediction of Myocardial Recovery

All 13 patients completed the dobutamine stress protocol without complication. The mean double product at rest was 8745 , and this increased to 9923 at $5 \mu \mathrm{g} \cdot \mathrm{kg}^{-1} \cdot \mathrm{min}^{-1}$ dobutamine and to 10,322 at $10 \mu \mathrm{g} \cdot \mathrm{kg}^{-1} \cdot \mathrm{min}^{-1}$ dobutamine. Regional strain values are summarized in Table 2. Regional strain values with dobutamine stress were significantly different from strain values at rest. Low-dose dobutamine stress testing with myocardial strain analysis predicted recovery of function in $64 \%$ to $77 \%$ of regions (Table 3 ). More specifically, when choosing cutoff points to determine sensitivity, specificity, and likelihood ratios for prediction of myocardial recoverability with dobutamine stress, the maximal sum of sensitivity and specificity resulted in the best specificity with modest sensitivity (Table 4). Alternatively, when sensitivity was set at $80 \%$ or $90 \%$, the specificity decreased, but the likelihood ratios were similar.

\section{Discussion}

The results of this study demonstrate the feasibility of predicting recovery of myocardial function after $\mathrm{CABG}$ tissue-tagging MR imaging to determine changes in myocardial strain with dobutamine challenge. Among 3 strain parameters tested, minimum principal strain had the highest sensitivity and specificity at both 5 and $10 \mu \mathrm{g} \cdot \mathrm{kg}^{-1}$. $\min ^{-1}$ dobutamine, as evidenced by the likelihood ratios. Circumferential strain was slightly less accurate than minimum principal strain at each stress level and sensitivity, whereas radial strain was more variable than either minimum principal strain or circumferential strain.

Previous studies from our laboratory have measured decreased regional circumferential strain in patients with coronary artery disease, followed by improvement in 
TABLE 2. Regional strain values at rest and with dobutamine stress*

\begin{tabular}{|c|c|c|c|c|c|c|c|c|c|}
\hline \multirow[b]{2}{*}{ Regions } & \multicolumn{3}{|c|}{ Minimum principal strain } & \multicolumn{3}{|c|}{ Circumferential strain } & \multicolumn{3}{|c|}{ Radial strain } \\
\hline & Rest & $5 \mu \mathrm{g}$ & $10 \mu \mathrm{g}$ & Rest & $5 \mu \mathrm{g}$ & $10 \mu \mathrm{g}$ & Rest & $5 \mu \mathrm{g}$ & $10 \mu \mathrm{g}$ \\
\hline AS & $15 \pm 0.3$ & $15 \pm 0.5$ & $11 \pm 0.4$ & $8 \pm 0.6$ & $10 \pm 0.9$ & $7 \pm 0.5$ & $9 \pm 0.9$ & $16 \pm 1.1$ & $9 \pm 0.8$ \\
\hline ANT & $15 \pm 0.5$ & $17 \pm 0.6$ & $14 \pm 0.5$ & $12 \pm 0.5$ & $15 \pm 0.6$ & $11 \pm 0.6$ & $11 \pm 0.7$ & $15 \pm 0.6$ & $12 \pm 0.9$ \\
\hline $\mathrm{AL}$ & $15 \pm 0.5$ & $18 \pm 0.6$ & $15 \pm 0.7$ & $12 \pm 0.5$ & $16 \pm 0.5$ & $12 \pm 0.7$ & $24 \pm 0.8$ & $21 \pm 0.7$ & $26 \pm 0.8$ \\
\hline PL & $12 \pm 0.5$ & $15 \pm 0.5$ & $12 \pm 0.7$ & $11 \pm 0.5$ & $13 \pm 0.6$ & $10 \pm 0.8$ & $28 \pm 0.9$ & $25 \pm 0.7$ & $34 \pm 1.9$ \\
\hline POST & $11 \pm 0.6$ & $11 \pm 0.6$ & $10 \pm 0.6$ & $10 \pm 0.6$ & $9 \pm 0.6$ & $8 \pm 0.5$ & $21 \pm 0.6$ & $30 \pm 1.4$ & $30 \pm 1.5$ \\
\hline PS & $14 \pm 0.4$ & $14 \pm 0.6$ & $12 \pm 0.4$ & $13 \pm 0.4$ & $12 \pm 0.6$ & $9 \pm 0.4$ & $19 \pm 0.5$ & $25 \pm 1.2$ & $19 \pm 0.9$ \\
\hline
\end{tabular}

$A S$, Anteroseptal; $A N T$, anterior; $A L$, anterolateral; $P L$, posterolateral; $P O S T$, posterior; $P S$, posteroseptal. * Mean \pm standard deviation.

myocardial strain after surgical revascularization. ${ }^{16}$ Subsequently, using tissue-tagged MR imaging strain analysis, we have quantitated the changes in minimum principal strain during dobutamine stress in patients with normal cardiac function and patients with dilated cardiomyopathy. ${ }^{17}$ The present study was the next step in evaluating tissue-tagged MR imaging with myocardial strain analysis as a potentially clinically applicable quantitative test for LV function and detection of myocardial viability.

The accuracy for detection of recoverability of myocardium after revascularization in our study is slightly less than previously identified with other MR parameters of myocardial function. Baer and colleagues ${ }^{3}$ found that an increase in systolic wall thickening of greater than $2 \mathrm{~mm}$ with dobutamine stress or diastolic wall thickness of greater than 5.5 $\mathrm{mm}$ had an accuracy of $82 \%$ and $79 \%$ for predicting recovery of function after revascularization, respectively. Wellnhofer and associates ${ }^{6}$ found that visual improvements in regional wall motion with dobutamine stress had an accuracy of $85 \%$ for predicting recovery of LV function after revascularization. In these studies the left ventricle was divided into either 8 or 16 segments per image, resulting in more data points and smaller regions with less heterogeneity. We chose to divide the left ventricle into the American Society of Echocardiography-recommended 16-segment model for LV segmentation, resulting in 6 segments per image. ${ }^{18}$ This might have resulted in regions with variable amounts of scarred

TABLE 3. Odds ratios and areas under the ROC curve for myocardial strain parameters for prediction of myocardial recoverability with dobutamine stress

\begin{tabular}{lrcc} 
Strain/stress level & $\boldsymbol{P}$ value & Odds ratio $(\mathbf{9 5 \%}$ Cl) & Area under ROC curve \\
\hline MPS, $5 \mu \mathrm{g}$ & $<.001$ & $1.16(1.06-1.27)$ & 0.75 \\
MPS, $10 \mu \mathrm{g}$ & $<.001$ & $1.14(1.06-1.23)$ & 0.75 \\
CS, $5 \mu \mathrm{g}$ & .002 & $1.14(1.05-1.23)$ & 0.72 \\
CS, $10 \mu \mathrm{g}$ & .007 & $1.10(1.03-1.17)$ & 0.70 \\
RS, $5 \mu \mathrm{g}$ & $<.001$ & $1.09(1.04-1.14)$ & 0.77 \\
RS, $10 \mu \mathrm{g}$ & .030 & $1.04(1.0-1.08)$ & 0.64
\end{tabular}

ROC, Receiver operating characteristic; $C l$, confidence interval; MPS, minimum principal strain; $C S$, circumferential strain; $R S$, radial strain. and stunned myocardium. This heterogeneity within regions might explain the slightly decreased accuracy of myocardial strain analysis for the prediction of recoverability. Furthermore, it is likely that the full potential of the quantitative tissue-tagging approach is not represented in this study. Our analysis was limited to 2 dimensions for reasons of practicality. Accordingly, we could not account for through-plane motion. Thus far, placement of tag points is only semiautomated, making this analysis a very labor-intensive exercise. With software advances, more complex and comprehensive studies could be performed, including the addition of the longitudinal dimension to the analysis. True 3-dimensional assessment of the effect of dobutamine challenge on the apical segments could then be included in the determination of recoverability and would likely increase the accuracy of the tool.

TABLE 4. Sensitivities, specificities, and likelihood ratios for myocardial strain parameters with dobutamine stress for the prediction of myocardial recoverability after coronary artery bypass grafting

\begin{tabular}{lrrrrr}
\hline \multicolumn{1}{c}{ Strain/stress level } & $\begin{array}{c}\text { Cutoff } \\
\text { point }\end{array}$ & Sensitivity & Specificity & Sum & Likelihood \\
ratio
\end{tabular}


Patients with viable myocardium on noninvasive testing demonstrate improved survival with revascularization compared with those receiving medical therapy. ${ }^{19,20}$ One metaanalysis demonstrated an $80 \%$ relative risk reduction in death with revascularization compared with medical therapy when viability was present, whereas there was no benefit when viability was not present. ${ }^{19}$ The best test to determine myocardial viability has yet to be determined. Dobutamine echocardiography is the most widely used clinical tool to evaluate myocardial viability, with published sensitivities and specificities approximating $80 \% .^{21}$ Nuclear medicine studies, including positron emission tomography, technetium-99m sestamibi, and thallium-201 studies, have similar sensitivity when compared with dobutamine echocardiography, but the specificity appears slightly less. ${ }^{21}$ MR imaging is an intriguing tool to evaluate myocardial viability because of its quantitative nature, improved spatial resolution, and potential to evaluate function, perfusion, and anatomy with a single test. The current study suggests that low-dose dobutamine cardiac MR imaging with strain analysis has the potential to accurately predict myocardial viability. Future study incorporating quantitative analysis, perfusion, and delayed contrast enhancement might improve the accuracy of predicting viable myocardium by using MR imaging.

The current study is limited by the small number of patients and the lack of consistent comparative viability assessments using echocardiography or nuclear techniques. Unfortunately, the practicalities of clinical practice in the current health care environment present significant logistic challenges. Only 9 patients underwent clinically indicated viability testing, of whom 3 patients had preoperative dobutamine stress echocardiography, whereas the others underwent sestamibi or positron emission tomographic scans. With only this small number of studies, no useful comparisons could be made. Additionally, 7 patients were excluded from the final analysis because of equipment failure or personal reasons. Despite these limitations, we believe the current study supports the continued investigation of tissuetagged MR imaging with strain analysis as an option for assessing myocardial recoverability quantitatively in patients requiring coronary revascularization.

\section{References}

1. Eletteriades JA, Tolis G, Levi E, Mills LK, Zaret BL. Coronary artery bypass grafting in severe left ventricular dysfunction: excellent survival with improved ejection fraction and functional state. J Am Coll Cardiol. 1993;22:1411-7.

2. Detre K, Peduzzi P, Murphy M, et al. Effect of bypass surgery on survival in patients in low and high risk subgroups delineated by the use of simple clinical variables. Circulation. 1981;63:1329-38.
3. Baer FM, Theissen P, Schneider CA, Voth E, Sechtem U, Schicha H, et al. Dobutamine magnetic resonance imaging predicts contractile recovery of chronically dysfunctional myocardium after successful revascularization. J Am Coll Cardiol. 1998;31:1040-8.

4. Klow NE, Smith HJ, Gullestad L, Seem E, Endresen K. Outcome of bypass surgery in patients with chronic ischemic left ventricular dysfunction. Predictive value of MR imaging. Acta Radiol. 1997;38:76-82.

5. Baer FM, Voth E, Schneider CA, Theissen P, Schicha H, Sechtem U. Comparison of low-dose dobutamine-gradient-echo magnetic resonance imaging and positron emission tomography with [18F]fluorodeoxyglucose in patients with chronic coronary artery disease. A functional and morphological approach to the detection of residual myocardial viability. Circulation. 1995;91:1006-15.

6. Wellnhofer E, Olariu A, Klein C, Grafe M, Wahl A, Fleck E, Nagel E. Magnetic resonance low-dose dobutamine test is superior to scar quantification for the prediction of functional recovery. Circulation. 2004; 109:2172-4.

7. Nagel E, Lehmkuhl HB, Bocksch W, Klein C, Vogel U, Frantz E, et al. Noninvasive diagnosis of ischemia-induced wall motion abnormalities with the use of high-dose dobutamine stress MRI: comparison with dobutamine stress echocardiography. Circulation. 1999;99:763-70.

8. Sandstede JJ, Bertsch G, Beer M, et al. Detection of myocardial viability by low-dose dobutamine cine MR imaging. Magn Reson Imaging. 1999; $17: 1437-43$

9. Kuijpers D, Ho KY, van Dijkman PR, Vliegenthart R, Oudkerk M. Dobutamine cardiovascular magnetic resonance for the detection of myocardial ischemia with the use of myocardial tagging. Circulation. 2003;107:1592-7.

10. Gerber BL, Garot J, Bluemke DA, Wu KC, Lima JAC. Accuracy of contrast-enhanced magnetic resonance imaging in predicting improvement of regional myocardial function in patients after acute myocardial infarction. Circulation. 2002;106:1083-9.

11. Ungacta FF, Davila-Roman VG, Moulton MJ, et al. MRI-radiofrequency tissue tagging patients with aortic insufficiency before and after operation. Ann Thorac Surg. 1998;65:943-50.

12. Axel L. Noninvasive measurement of cardiac strain with MRI. Adv Exp Med Biol. 1997;430:249-56.

13. Fung YC. A first course in continuum mechanics. 3rd ed. Englewood Cliffs, NJ: Prentice-Hall; 1994.

14. Szabo BA, Babuska I. Finite element analysis. New York: Wiley; 1991.

15. Hosmer DW, Lemeshow S. Applied logistic regression. 2nd ed. New York: John Wiley and Sons; 2000.

16. Maniar HS, Cupps BP, Potter DD, et al. Ventricular function after coronary artery bypass grafting: evaluation by magnetic resonance imaging and myocardial strain analysis. $J$ Thorac Cardiovasc Surg. 2004;128:76-82.

17. Moustakidis P, Cupps BP, Pomerantz BJ, et al. Noninvasive, quantitative assessment of left ventricular function in ischemic cardiomyopathy. J Surg Res. 2004;116:187-96.

18. Schiller NB, Shah PM, Crawford M, et al. Recommendations for quantitation of the left ventricle by two-dimensional echocardiography American Society of Echocardiography Committee on Standards, Subcommittee on Quantitation of Two-dimensional Echocardiograms. J Am Soc Echocardiogr. 1989;2:358-67.

19. Bax JJ, van der Wall EE, Harbinson M. Radionuclide techniques for the assessment of myocardial viability and hibernation. Heart. 2004;90: 26-33.

20. Allman KC, Shaw LJ, Hachamovitch R, Udelson JE. Myocardial viability testing and impact of revascularization on prognosis in patients with coronary artery disease and left ventricular systolic dysfunction: a meta-analysis. J Am Coll Cardiol. 2002;39:1151-8.

21. Bax JJ, Poldermans D, Elhendy A, Boersma E, Rahimtoola SH. Sensitivity, specificity, and predictive accuracies of various noninvasive techniques for detecting hibernating myocardium. Curr Probl Cardiol. 2001;26:141-86. 